\title{
Effects of Solvent Molecules on the Interlayer Spacing of Graphene Oxide
}

\author{
Liyan Liu ${ }^{1} \cdot$ Ruifeng Zhang ${ }^{1} \cdot$ Ying Liu $^{1} \cdot$ Haonan Zhu ${ }^{1} \cdot$ Wei Tan ${ }^{1} \cdot$ Guorui Zhu ${ }^{1} \cdot$ Yang Wang $^{1}$
}

Received: 8 March 2018 / Revised: 14 April 2018 / Accepted: 22 April 2018 / Published online: 23 May 2018

(c) The Author(s) 2018

\begin{abstract}
Graphene oxide (GO) contains numerous functional groups that facilitate the intercalation of polar solvents. The properties and applications of GO are closely related to its interlayer spacing. We report on the changes in the interlayer spacing of GO after the adsorption of water molecules and the polar organic solvents $\mathrm{C}_{2} \mathrm{H}_{6} \mathrm{O}_{2}(\mathrm{EG}), \mathrm{C}_{3} \mathrm{H}_{7} \mathrm{NO}(\mathrm{DMF}), \mathrm{C}_{5} \mathrm{H}_{9} \mathrm{NO}$ (NMP). Experiments were conducted to investigate the variations in the functional groups and structure of $\mathrm{GO}$ after solvent adsorption, and they play a vital role in modeling and verifying the results of molecular dynamics simulation. The most stable GO structures are obtained through molecular dynamics simulation. The expansion of the interlayer spacing of GO after the adsorption of monolayer solvent molecules corresponds to the minimum three-dimensional size of the solvent molecules. The spatial arrangement of solvent molecules also contributes to the changes in interlayer spacing. Most adsorbed molecules are oriented parallel to the carbon plane of GO. However, as additional molecules are adsorbed into the interlaminations of GO, the adsorbed molecules are oriented perpendicular to the carbon plane of GO, and a large space forms between two GO interlayers. In addition, the role of large molecules in increasing interlayer spacing becomes more crucial than that of water molecules in the adsorption of binary solvent systems by GO.
\end{abstract}

Keywords Graphene oxide · Interlayer spacing · Solvent adsorption

\section{Introduction}

The layered structure and large area of graphene oxide (GO) facilitate the intercalation and adsorption of polar solvent molecules through covalent or noncovalent approaches [1]. The dispersibility and the electronic, optical, and mechanic properties of GO are affected by its interlaying spacing [2, 3]. These properties, in turn, are responsible for the extensive applications of GO. Research on the effects of some solvent molecules on the interlayer spacing of GO will help guide the further applications of this material.

Several experiments and simulations have been executed to study the structure of GO and the behavior of water confined in GO. Rezania et al. [4] investigated the hydration of single-layered GO and revealed through X-ray diffraction (XRD) and neutron scattering analysis that interlayer spacing of GO expands with relative humidity. Through molecular

Yang Wang

yangwang2017@tju.edu.cn

1 School of Chemical Engineering and Technology, Tianjin University, Tianjin 300350, China dynamics (MD) simulation, Zhou et al. [5] demonstrated that the stacking pattern of confined water molecules in GO is affected by the distance between neighboring layers. Moreover, water molecules present in different regions of GO display different behaviors. For example, regions close to the GO surface are isolated, and other regions distant from the GO surface are surrounded by water molecules [6].

The effect of polar solvent molecules on the interlayer spacing of GO is more drastic than that of water molecules. The interlayer spacing of GO upon maximum adsorption changes when GO is exposed to an atmosphere saturated with different polar solvents. This behavior provides evidence that small molecules are easily intercalated into the GO interlaminations [7]. Solvent molecules in vapor, liquid, and solid phases can be intercalated into GO. The intercalated molecules occupy the expanded interlayer spacing of GO along the $c$-axis. Some researchers have connected the increase in interlayer spacing with the number and size of the intercalated solvent molecules $[8,9]$. These researchers described molecular size by using only one parameter. In reality, however, the three-dimensional sizes of molecules vary greatly. To the best of our knowledge, research on the 
interlayer spacing of different types of solvents that coexist in GO interlaminations does not exist. MD is an effective method for controlling the number of solvent molecules and for visualizing the simultaneous adsorption of two kinds of molecules into GO.

This work focused on the intercalation of water and typical polar solvent molecules into the GO interlaminations. The interlayer spacing values of GO after the intercalation of one or two kinds of solvent molecules under ambient temperature were quantified through MD. Experiments were conducted to determine the validity of the simulated model and to ensure the accuracy of simulation results. The increase in the interlayer spacing of GO after solvent adsorption depends on the molecular size of the solvent. Instead of evaluating molecular size solely on the basis of diameter, as was done in a previous study [8], we calculated the three-dimensional size and it could be applied to longchain solvent molecules. The behavior of solvent molecules closely corresponded with the amount and size of the solvent molecules. In addition, we identified the molecules that dominate the increase in the interlayer spacing of GO when two different types of molecules are intercalated into GO.

\section{Methods}

\section{Experimental Methods}

\section{Preparation of Materials}

GO was prepared from natural graphite powder through a modified Hummers' method [10, 11]. A stable suspension was obtained after the products were purified by using a novel electrodialysis system that we have previously reported [12]. Then, a vacuum freeze drier (FD-1B-50, Shanghai, China) was used to remove water molecules from the suspension. Finally, the dried GO was milled into powder.

Ethylene glycol (EG), N,N-dimethylformamide (DMF), $\mathrm{N}$-methyl-2-pyrrolidone (NMP) are excellent solvents that strongly interact with GO $[2,13]$. Deionized water, EG (AR), DMF (AR), and NMP (AR) were used as solvents in this study and were purchased from Yuan Li Chemical Co., Ltd, China.

\section{Preparation of GO-Adsorbing Solvent Molecules}

One gram of GO powder was placed on filter paper. The filter paper with GO powder was then placed on a beaker containing $120 \mathrm{~mL}$ of water, EG, DMF, or NMP, respectively. Given that the polar solvents volatilized and easily penetrated through filter paper, the solvent molecules entered the interlaminations of GO and bonded with the functional groups of GO. The amounts of solvent molecules adsorbed in GO varied with time $t(\mathrm{~h})$. Samples were taken at different time points for testing until GO achieved its adsorption saturation state.

To verify that solvents existed in the GO interlamination, samples were examined through Fourier transform infrared spectroscope (FTIR, Nicolet 6700, USA). Samples were mixed with $\mathrm{KBr}$ powder and pelletized before the scan was performed over the wave number of $4000-500 \mathrm{~cm}^{-1}$. XRD patterns were recorded on a high-resolution X-ray diffractometer (Bruker, Germany) under a scan rate of $3 \% \mathrm{~min}$ in a $2 \theta$ range of $5^{\circ}-30^{\circ}$ with $\mathrm{Cu} \mathrm{K} \alpha$ radiation $(\lambda=1.5418 \AA)$ at $40 \mathrm{kV}$ and $40 \mathrm{~mA}$.

NMP was the most structurally complex solvent among the solvents tested in this study and possessed all of the functional groups possessed by the other three solvents. NMP was selected as the representative solvent for intercalation into the GO interlaminations for the comparison between the experimental and simulated interlayer spacing values. The weight of dry GO is $m_{0}\left(m_{0}=1 \mathrm{~g}\right)$, and that with NMP solvent is $m_{t}$. The values of $m_{t}$ were recorded at time $t(\mathrm{~h})$ until the GO reached its adsorption saturation state. The percentage of NMP solvent adsorbed into layers was quantified as:

content $=\frac{m_{t}-m_{0}}{m_{t}} \times 100 \%$.

\section{Molecular Dynamics}

The composition [14] applied in the following simulations was $\mathrm{C}_{8} \mathrm{O}_{2}(\mathrm{OH})_{2}$. An example of atomic configuration is presented in Fig. 1. The unit cell consisted of three platelets with an initial interlayer spacing of $15 \AA$ [15]. Periodic boundary conditions were applied in three directions, and the size of the original simulation box was $39.36 \AA \times 19.67 \AA \times 45 \AA$. Different numbers of solvent molecules were adsorbed in the cavity formed between two neighboring platelets.

Geometry optimization and MD were carried out in the Forcite Module executed in Materials Studio (MS) software. MD was conducted with a Dreiding [15] forcefield and Qeq charge equilibrium equation [16]. Dreiding is the only forcefield that considers interlayer interactions among van

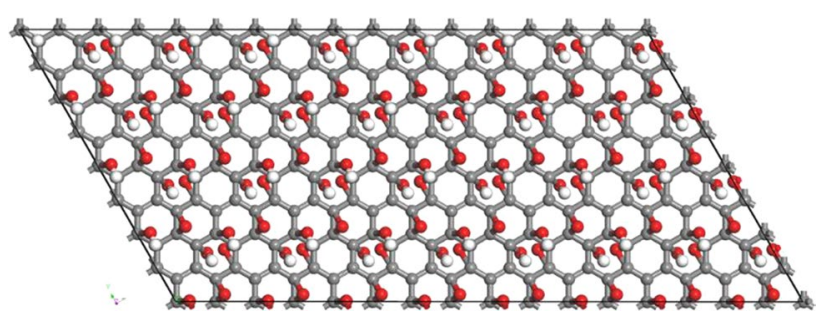

Fig. 1 Atomic configuration of hydroxyl and epoxide groups on the surface of GO. Gray, white, and red spheres represent carbon, hydrogen, and oxygen atoms, respectively 
der Waals (VDWs), hydrogen bonding (H-bond), and electrostatic in MS [17]. VDWs nonbonded interactions were described on the basis of 12-6 Lennard-Jones potential [18, 19], at the cutoff distance of $20 \AA$. H-bond interactions were described as 12-10 exponential functions [17]. GO-solvent molecule structures were first equilibrated through 50,000 iterations of energy minimization by adjusting atom coordinates. A fine convergence tolerance was reached with the conjugate gradient algorithm. After optimization, cell energy reached the minimum value, and the system was the most stable. To ensure the equilibration of the GO-solvent molecular system, the system was switched to MD. The structure was calculated using a canonical ensemble (NVT) condition for $5 \mathrm{~ns}$ at a time step of $1 \mathrm{fs}$. The temperature of the heat bath was $298 \mathrm{~K}$ and was controlled with a Nose thermostat.

\section{Results and Discussion}

\section{Characterization of the Prepared Samples}

\section{FTIR Characterization}

The FTIR spectrum was used to characterize the changes in the chemical bonds and functional groups of GO after solvent adsorption. As shown in Fig. 2, all the FTIR spectra of GO-solvents exhibit representative peaks at $\sim 3435$, $\sim 1640, \sim 1400$, and $\sim 1058 \mathrm{~cm}^{-1}$ that correspond to the $\mathrm{O}-\mathrm{H}$ stretching vibration, $\mathrm{C}=\mathrm{O}$ stretching vibration, $\mathrm{O}-\mathrm{H}$ deformation vibration, and epoxy $\mathrm{C}-\mathrm{O}$ stretching vibration, respectively. As previously described, hydroxyl, carboxyl, and epoxy groups exist in GO planes. In addition, the FTIR spectrum of GO-NMP exhibited peaks at 2926 and $2852 \mathrm{~cm}^{-1}$ and that of GO-DMF and GO-EG exhibited peaks at 2928 and $2871 \mathrm{~cm}^{-1}$, respectively. The peaks at $\sim 2927$ and $\sim 2861 \mathrm{~cm}^{-1}$ could be attributed to the $-\mathrm{CH}_{3}$ and $-\mathrm{CH}_{2}$ stretching vibration, respectively [20]. The FTIR spectrum of GO-DMF presented a new peak at $1437 \mathrm{~cm}^{-1}$ attributed to $\mathrm{C}-\mathrm{N}$ stretching vibration. In addition to the functional groups that exist in GO, GO-NMP contains $-\mathrm{CH}_{3}$ and $-\mathrm{CH}_{2}$ groups, GO-DMF contains $-\mathrm{CH}_{3}$ and $\mathrm{C}-\mathrm{N}$ groups, and $\mathrm{GO}-\mathrm{EG}$ contains $-\mathrm{CH}_{2}$ groups. The appearance of new peaks confirmed that solvent molecules have intercalated in GO.

\section{XRD Analysis of GO-Solvents}

Interlayer spacing plays an important role in determining the structure and crystallinity of carbon materials. The increase in the interlayer spacing of GO is correlated with the amount of solvents that have intercalated in the interlaminations and is calculated using the Bragg equation, which is deduced from the position of (001) reflection in the XRD patterns of
Fig. 2 FTIR spectra of GO upon the adsorption of different solvent molecules. a Water, b EG, $\mathbf{c}$ DMF, and $\mathbf{d}$ NMP
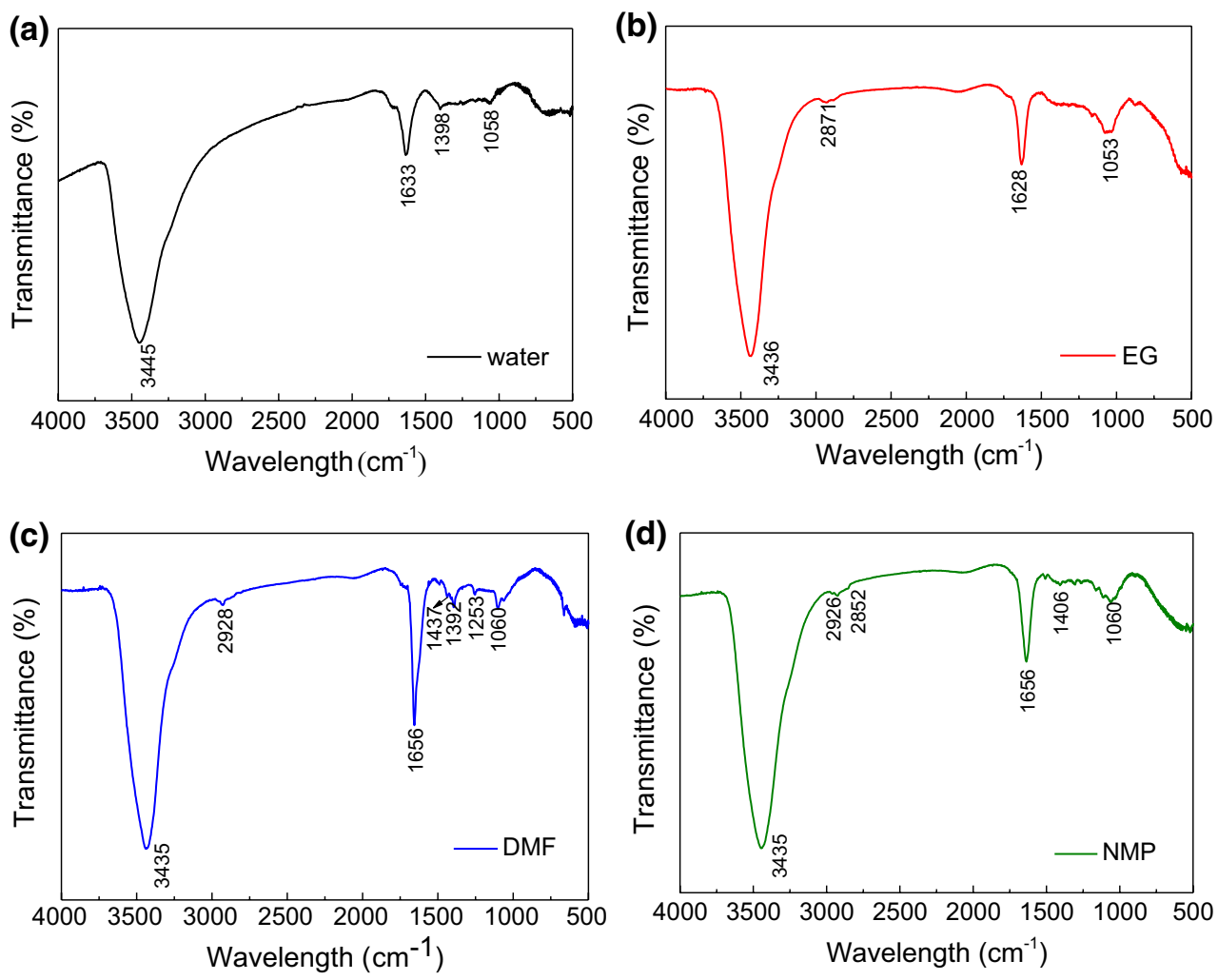
GO-solvent systems. Notably, as a result of the interstratification and nonuniform hydration of GO interlayers, the $d$ (001) of the Hummers GO changes regularly [4].

Examples of the XRD patterns of the GO-solvent systems under ambient temperature are shown in Fig. 3. Two characteristic peaks appeared in the spectra of the GO-EG, GO-DMF, GO-NMP systems after 24,1 , and 8 h of adsorption. The appearance of these peaks was caused by the adsorption of monolayer solvents into the GO interlaminations. The three-dimensional sizes of DMF and NMP molecules are nonuniform, which will slightly change the delamination behavior of neighboring layers in the presence of excess DMF and NMP molecules.

\section{Verification}

The GO-NMP interlayer spacing values obtained through experiments and simulations are shown in Fig. 4. The simulated interlayer spacing values are very close to the experimental values. The simulated and experimental interlayer spacing of GO increased to $13 \AA$. Except for the point of NMP content at $0 \%$, the deviation is less than $10 \%$ through (a)

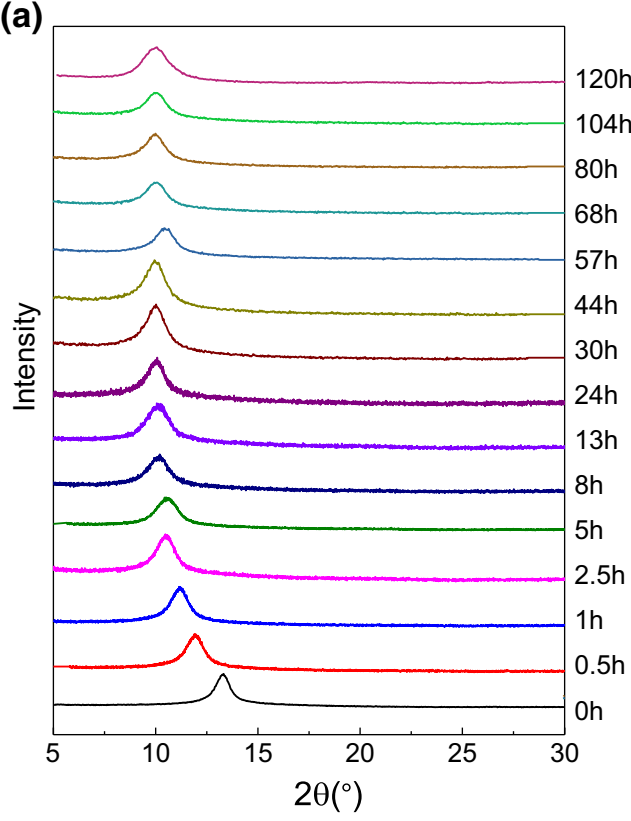

(c)

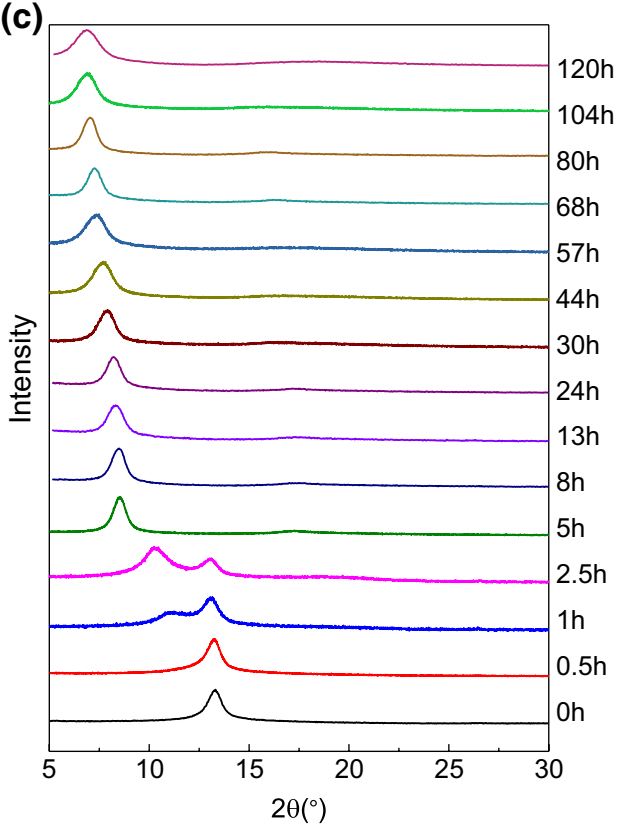

(b)

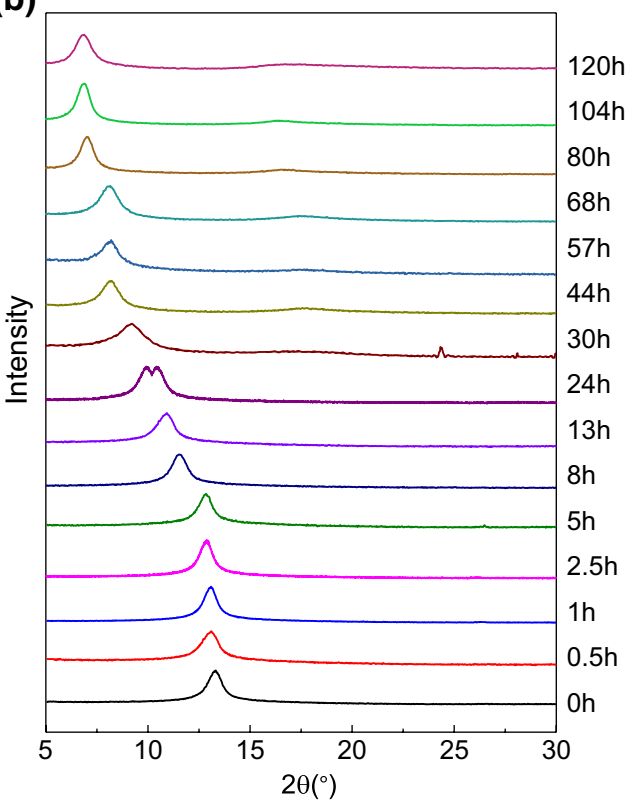

(d)

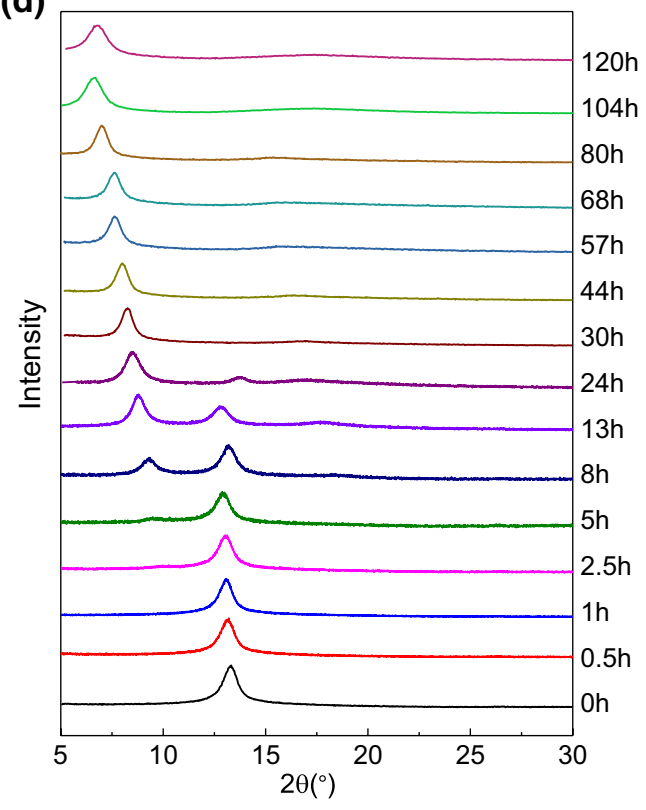

Fig. 3 XRD patterns of GO after the adsorption of a water, $\mathbf{b}$ EG, $\mathbf{c}$ DMF, and $\mathbf{d}$ NMP 


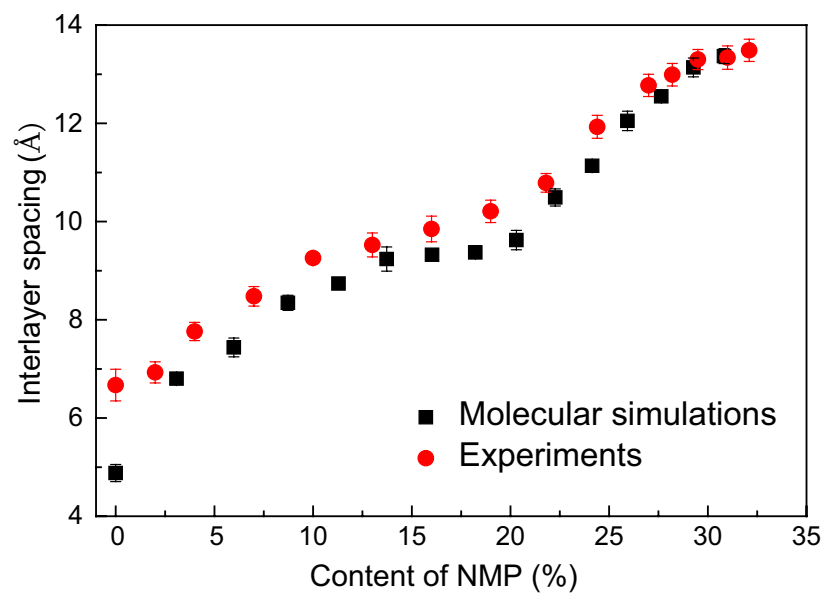

Fig. 4 Experimental and simulated interlayer spacing values of GONMP

all the researched content of NMP. The deviation between experimental and simulated values can be explained as follows: Water molecules bond tightly with GO layers when the water content is low and cannot be removed even after prolonged drying [21]. Thus, the dried GO samples contained irremovable moisture. Furthermore, the GO samples simultaneously adsorbed moisture from the air while adsorbing solvent molecules. These two moisture components will slightly increase the experimental interlayer spacing. XRD results showed that interlayer spacing increased by a maximum of $0.85 \AA$ when dry GO powder was placed in an atmosphere with a relative humidity of $58.2 \%$. Thus, the interlayer spacing obtained through MD is closer to the ideal state than that obtained through experiments. In conclusion, all the settings and procedures used in the MD simulation of the GO structure are suitable and can be used in the following calculations.

\section{Relationship Between the Interlayer Spacing of GO and the Size of Solvent Molecules}

As previously stated, the diffraction angle of the first characteristic peak decreases with time. This behavior proves that the interlayer spacing increases with solvent adsorption. The detailed simulated results for the interlayer spacing of GO after the intercalation of different solvent molecules are shown in Fig. 5. The interlayer spacing of GO will expand given that the free space will be separated by GO layers and will become filled with solvent molecules [22]. Consistent with the result presented by [23], the interlayer spacing of GO will expand by different degrees with the addition of solvent molecules to the system.

Some researchers have correlated the interlayer spacing value of GO with molecular diameter [8]. This relationship can effectively describe the intercalation of small

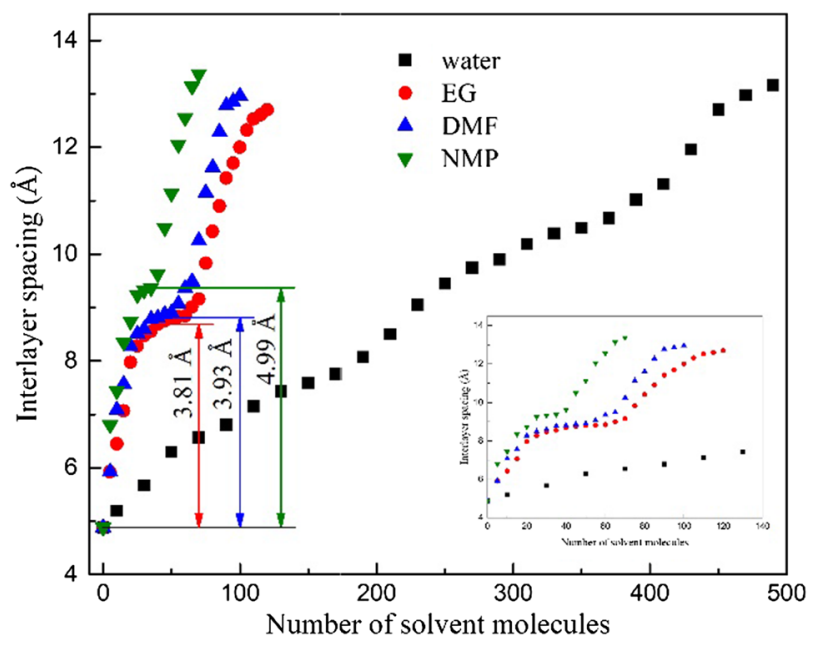

Fig. 5 Interlayer spacing of GO after the adsorption of water, EG, DMF, or NMP. The figure in the lower right corner shows the enlarged results of these solvents in GO layers

molecules, such as water. However, the results show large deviations of long-chain molecules into GO. In this work, the three-dimensional molecular sizes of four kinds of solvents were determined. A solvent molecule was placed in a rectangular box with a minimum volume. The length, width, and height of the rectangular box were defined as solvent molecular size. The three-dimensional structures (length $\times$ width $\times$ height) of water, EG, DMF, and NMP are shown in Fig. 6. Three-dimensional molecular sizes of the solvents follow the order of water $<\mathrm{EG}<\mathrm{DMF}<\mathrm{NMP}$.

The maximum number of solvent molecules adsorbed by GO is related to the molecular size of the solvent. This relationship has been demonstrated in other carbon materials [24]. Our simulation results showed that the maximum numbers of water, EG, DMF, and NMP molecules that can be adsorbed per unit cell of GO are 490, 120, 97, and 70, respectively. The hydrodynamic diameter of a water molecule is $\sim 2.7 \AA$ [25]. The intercalation of a water monolayer causes the lattice of GO to expand by $2.2-2.5 \AA$ [24]. The size of the DMF layer adsorbed by GO structure is $\sim 4.4 \AA$ [26]. An increase in interlayer spacing of $7.3 \AA$ corresponds to the intercalation of two DMF layers into GO interlamination [9]. The increase in interlayer spacing caused by onelayer solvent molecules corresponds to the size parameter of the minimum three-dimensional molecule, as shown in Fig. 6.

The interlayer spacing curves of GO that has adsorbed EG, DMF, or NMP present plateaus, as shown in Fig. 5. When the interlayer spacing reaches the first plateau, monolayer solvent molecules will be intercalated into the interlamination. The variation in interlayer spacing from the beginning to the first plateau of the curve is similar to the variation shown by the minimum three-dimensional 
Fig. 6 Molecular size of a water, b EG, c DMF, and d NMP. Gray, white, blue, and red spheres represent $\mathrm{C}, \mathrm{H}, \mathrm{N}$, and $\mathrm{O}$ atoms, respectively
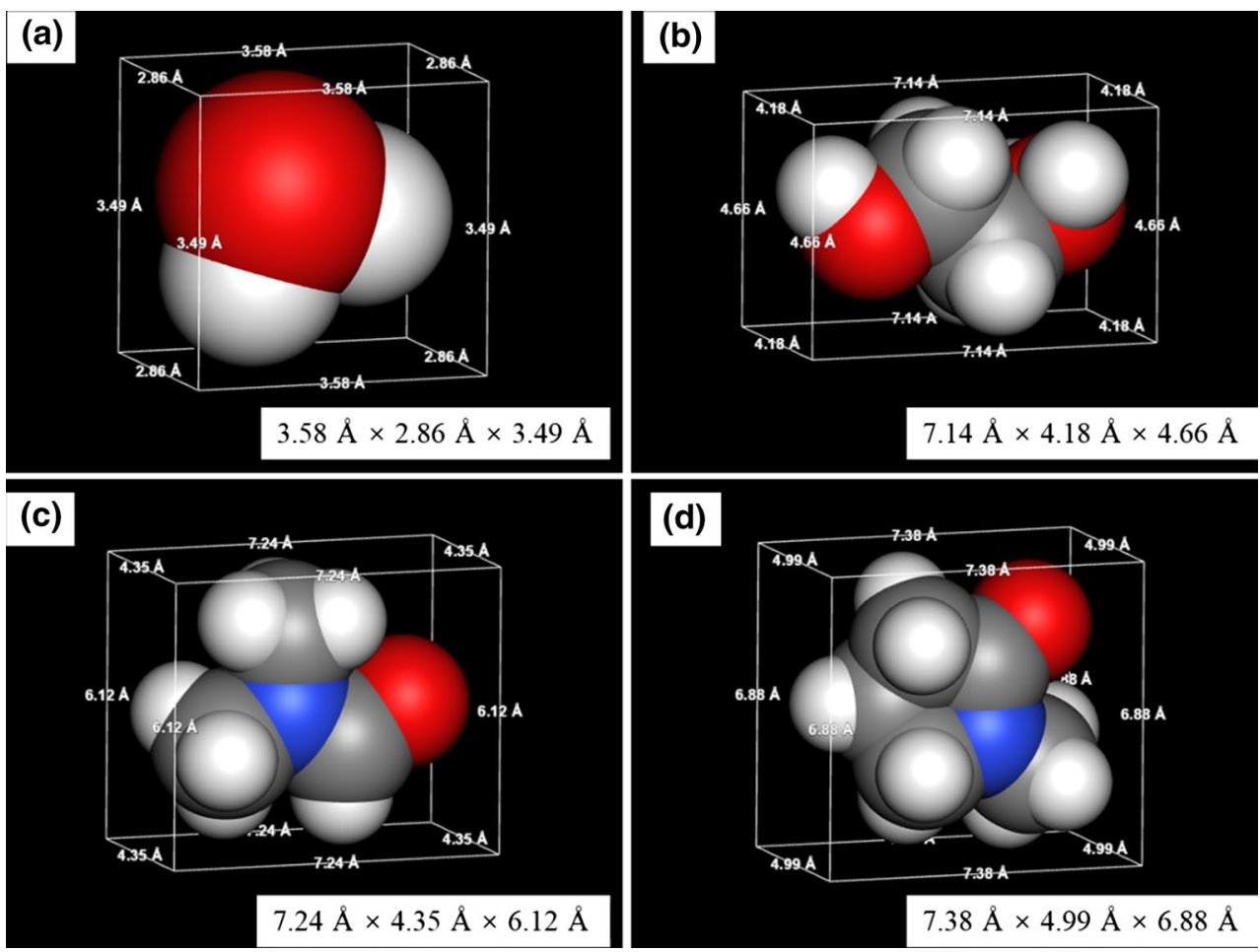

molecular size in Fig. 6. The expansion of interlayer spacing caused by the intercalation of one solvent molecule is consistent with the order of solvent molecular size. The plateau of water adsorption is unclear. The three-dimensional molecular size of water is more uniform than that of the other three solvents.

The value of interlayer spacing is affected by the spatial arrangement of solvents. As inferred from the structure of solvent molecules intercalated in the GO layer, the maximum plane of solvent molecules tends to be oriented parallel to the carbon plane of GO. Thus, the interaction between the functional groups of GO and solvent molecules is maximized. Taking NMP as an example, almost all the NMP molecules are horizontally oriented in the space formed by two layers, and GO has a tight structure when few solvent molecules are present in the interlamination (Fig. 7a). Several NMP molecules began to follow a vertical orientation while most molecules continued to follow a horizontal orientation when the number of solvent molecules increased in the interlamination (Fig. 7b).

\section{Interlayer Spacing Under the Coexistence of Water and Solvent Molecules in GO Layers}

To further demonstrate the effect of different molecular sizes on interlayer spacing, the simultaneous adsorption of water with EG, DMF, or NMP molecules into GO interlaminations was simulated. Two kinds of molecules (a) horizontal orientation

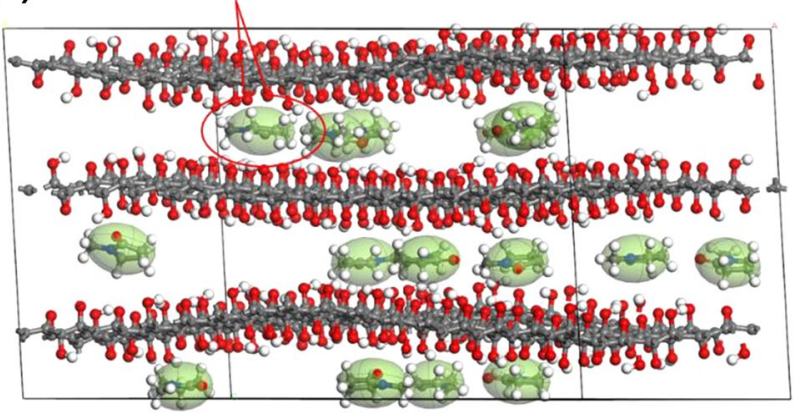

(b)

vertical orientation

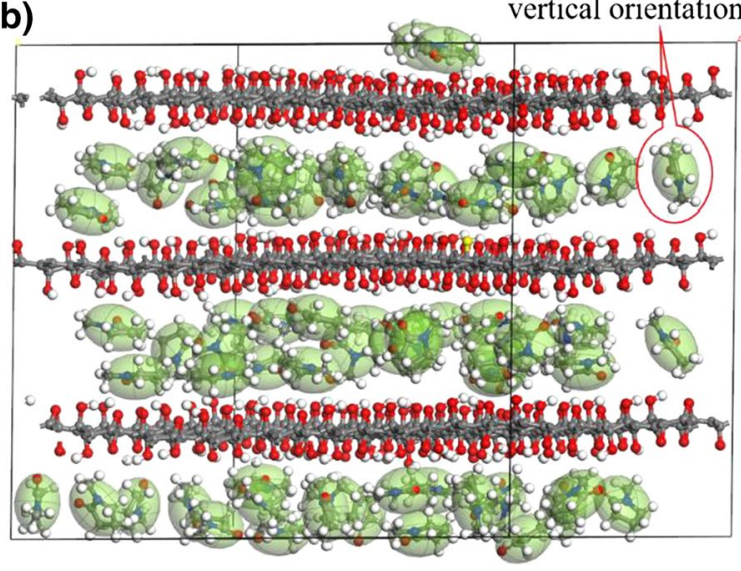

Fig. 7 Structure of NMP molecules in GO layer. a 15 NMP molecules, b 70 NMP molecules 
(a)

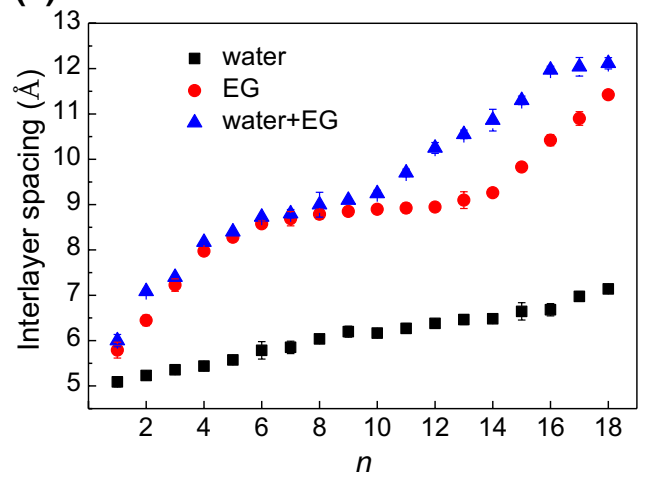

(b)

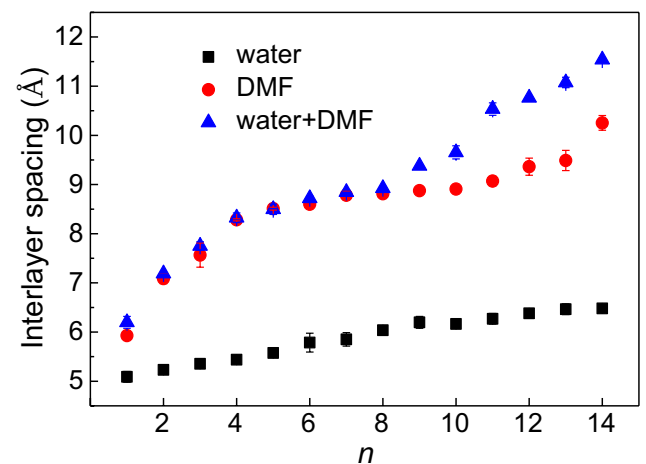

(c)

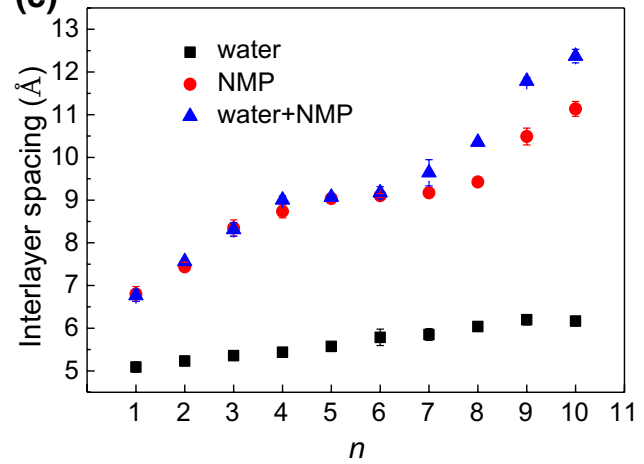

Fig. 8 Interlayer spacing of GO intercalated by unitary and binary solvent mixtures. a EG, b DMF, and c NMP

were simultaneously adsorbed to avoid the controlling effects observed in a previous study [27].

The confinement of one or two kinds of solvent molecules in the interlayer spacing of GO is presented in Fig. 8. The ratio of solvent molecules to $\mathrm{C}$ atoms of the GO plane was $5 n: 768$, and $n$ varied as solvent molecules were added to the system. Taking NMP as an example (Fig. 8c), it is shown that the interlayer spacing of the GO-water-NMP system approximated that of the GO-NMP system when $n$ was less than 7. Small molecules exert negligible effects on interlayer spacing, and water molecules exist in the void formed by large solvent molecules. The interlayer spacing value of GO after the adsorption of these two kinds of molecules was larger than that after the adsorption of any single molecules and was closer to that of the GO-NMP system when $n$ exceeded 7. The effect of small molecules must be considered, and water molecules are distributed close to GO surface. The functional groups of GO are hydrophilic and tightly bonded with water molecules. In conclusion, the structure of GO and the behavior of solvent molecules are influenced by the molecular size of the solvent.

\section{Conclusions}

The structure and interlayer spacing of GO after the intercalation of polar solvent molecules are determined through experiments and MD simulation.

The structure of GO after the intercalation of solvent molecules is distinctly related to the number and size of solvent molecules. It is particularly related to the minimum three-dimensional size of the solvent molecule. Almost all the solvent molecules are oriented horizontally in the interlamination space when interlayer spacing is relatively small. The solvent molecules begin to orient vertically with the introduction of additional solvent molecules to the GO interlamination. Binary solvent mixtures are adsorbed into the GO interlamination. Large molecules dominate the enlargement of interlayer spacing.

Therefore, the structure of GO after hydration/adsorption is related to the type, amount, and molecular size of the solvent.

Acknowledgements This work was supported by the National Natural Science Foundation of China (No. 21576188). The authors acknowledged the testing support from the Shandong Institute of Tianjin University and materials supply from the Key Laboratory of Carbon Materials, Institute of Coal Chemistry, Chinese Academy of Sciences. 
Open Access This article is distributed under the terms of the Creative Commons Attribution 4.0 International License (http://creativeco mmons.org/licenses/by/4.0/), which permits unrestricted use, distribution, and reproduction in any medium, provided you give appropriate credit to the original author(s) and the source, provide a link to the Creative Commons license, and indicate if changes were made.

\section{References}

1. Lee DW, De Los Santos VL, Seo JW et al (2010) The structure of graphite oxide: investigation of its surface chemical groups. J Phys Chem B 114(17):5723-5728

2. Ou E, Xie Y, Peng C et al (2013) High concentration and stable few-layer graphene dispersions prepared by the exfoliation of graphite in different organic solvents. RSC Adv 3(24):9490-9499

3. Omidvar A, RashidianVaziri MR, Jaleh B et al (2016) Metalenhanced fluorescence of graphene oxide by palladium nanoparticles in the blue-green part of the spectrum. Chin Phys B 25(11): 118102

4. Rezania B, Severin N, Talyzin AV et al (2014) Hydration of bilayered graphene oxide. Nano Lett 14(7):3993-3998

5. Zhou X, Duan Y, Wang L et al (2017) Layering of confined water between two graphene sheets and its liquid-liquid transition. Chin Phys B 26(10): 106401

6. Zokaie M, Foroutan M (2015) Comparative study on confinement effects of graphene and graphene oxide on structure and dynamics of water. RSC Adv 5(49):39330-39341

7. Barroso-Bujans F, Cerveny S, Alegría A et al (2010) Sorption and desorption behavior of water and organic solvents from graphite oxide. Carbon 48(11):3277-3286

8. You S, Luzan SM, Szabo T et al (2013) Effect of synthesis method on solvation and exfoliation of graphite oxide. Carbon 52:171-180

9. Klechikov A, Yu J, Thomas D et al (2015) Structure of graphene oxide membranes in solvents and solutions. Nanoscale 7(37):15374-15384

10. Hummers JWS, Offeman RE (1958) Preparation of graphitic oxide. J Am Chem Soc 80(6):1339

11. Kovtyukhova NI, Ollivier PJ, Martin BR et al (1999) Layer-bylayer assembly of ultrathin composite films from micron-sized graphite oxide sheets and polycations. Chem Mater 11(3):771-778

12. Tan W, Hao R, Liu Y et al (2016) Desalination of graphite oxide by electrodialysis. Desalination 385:53-57

13. Paredes JI, Villar-Rodil S, Martinez-Alonso A et al (2008) Graphene oxide dispersions in organic solvents. Langmuir 24(19):10560-10564
14. Szabó T, Berkesi O, Forgó P et al (2006) Evolution of surface functional groups in a series of progressively oxidized graphite oxides. Chem Mater 18(11):2740-2749

15. Devanathan R, Chase-Woods D, Shin Y et al (2016) Molecular dynamics simulations reveal that water diffusion between graphene oxide layers is slow. Sci Rep 6:29484

16. Kovář P, Pospíšil M, Káfuňková E et al (2010) Mg-Al layered double hydroxide intercalated with porphyrin anions: molecular simulations and experiments. J Mol Model 16(2):223-233

17. Mayo SL, Olafson BD, Goddard WA (1990) DREIDING: a generic force field for molecular simulations. J Phys Chem 94(26):8897-8909

18. Heinz H, Vaia RA, Farmer BL et al (2008) Accurate simulation of surfaces and interfaces of face-centered cubic metals using 12-6 and 9-6 Lennard-Jones potentials. J Phys Chem C 112(44):17281-17290

19. Wang MH, Xie YE, Chen YP (2017) Thermal transport in twisted few-layer graphene. Chin Phys B 26(11):116503

20. Cheng ZL, Li W, Wu PR et al (2017) A strategy for preparing modified graphene oxide with good dispersibility and transparency in oil. Ind Eng Chem Res 56(19):5527-5534

21. Medhekar NV, Ramasubramaniam A, Ruoff RS et al (2010) Hydrogen bond networks in graphene oxide composite paper: structure and mechanical properties. ACS Nano 4(4):2300-2306

22. Talyzin AV, Hausmaninger T, You S et al (2014) The structure of graphene oxide membranes in liquid water, ethanol and waterethanol mixtures. Nanoscale 6:272-281

23. Liu T, Chen YQ (2013) A molecular dynamics study of the swelling patterns of $\mathrm{Na} / \mathrm{Cs}$-montmorillonites and the hydration of interlayer cations. Chin Phys B 22(2):027103

24. Xiao X, Li F, Huang J et al (2012) Reduced adsorption of propanil to black carbon: effect of dissolved organic matter loading mode and molecule size. Environ Toxicol Chem 31(6):1187-1193

25. Devineau K, Bihannic I, Michot L et al (2006) In situ neutron diffraction analysis of the influence of geometric confinement on crystalline swelling of montmorillonite. Appl Clay Sci 31(1-2):76-84

26. You S, Luzan S, Yu J et al (2012) Phase transitions in graphite oxide solvates at temperatures near ambient. J Phys Chem Lett 3(7):812-817

27. Chen L, Shi G, Shen J et al (2017) Ion sieving in graphene oxide membranes via cationic control of interlayer spacing. Nature 550(7676):380-383 